\title{
Environmental toxic factors and clinical pattern of Parkinson's disease
}

\author{
Lilia Rotaru
}

\begin{abstract}
Laboratory of Functional Neurology, Diomid Gherman Institute of Neurology and Neurosurgery
\end{abstract}
Chisinau, the Republic of Moldova

\author{
Author's ORCID iD, academic degrees and contribution are available at the end of the article \\ Corresponding author - Lilia Rotaru, e-mail: liliarotaru@yahoo.com \\ Manuscript received August 02, 2021; revised manuscript October 01, 2021; published online October 12, 2021
}

\begin{abstract}
Background: Parkinson's disease (PD) - the most common neuro-degenerative movement disorder - is considered a result of a multifactorial pathogenic process modulated by cumulative and interactive effects of genes and exposures. An environmental exposure could enhance or create dopaminergic neurons vulnerability and increase PD risk. The purpose of the study was to find if excessive exposure to toxic environmental factors may influence clinical pattern of PD.

Material and methods: The study was conducted on 111 patients diagnosed with PD, study group being defined as PD exposed to toxins (33 patients), control group including PD patients without toxin exposure (78 patients). General epidemiological data and clinical data were recorded.

Results: Toxin exposure was found in 33 patients (29.73\%), more of them - men and rural residents. Toxin exposed PD patients had an insignificantly younger age. The most common disease phenotype in the study group was the akinetic-rigid phenotype $(64.7 \%, \mathrm{p}=0.040)$, bradykinesia being the most common sign at the disease onset $(57.6 \%, \mathrm{p}=0.008)$. Levodopa equivalent daily dose also was higher in the study group $(659.02 \pm 232.46, \mathrm{p}=0.042)$.

Conclusions: Excessive exposure to toxic environmental factors may influence the clinical pattern of PD. In this study the akinetic-rigid type was the predominant disease phenotype associated with toxin exposure. Doses needed for treatment were higher in PD patients exposed to toxins, as an indicator of a more severe motor impairment in this group.
\end{abstract}

Key words: Parkinson's disease, toxic environmental factors.

Cite this article

Rotaru L. Environmental toxic factors and clinical pattern of Parkinson's disease. Mold Med J. 2021;64(4):69-71. https://doi. org/10.52418/moldovan-med-j.64-4.21.13.

\section{Introduction}

Parkinson's disease is the most common neurodegenerative movement disorder with a prevalence of about $2 \%$ in the population over the age of 65 years [1].

PD has no known cause. The exposure of the human population to environmental contaminants is recognized as a significant contributing factor for the development of Parkinson's disease and other forms of parkinsonism. Evidence exists to suggest that age and gender and some environmental factors (pesticide exposure, occupation) are associated with the development of PD. Instead, tobacco use, and caffeine consumption are believed to be inversely associated to the development of PD, tobacco and black tea having a protective effect on age at onset in genetic PD LRRK2 [2, 3].

$\mathrm{PD}$ varies in age of symptom onset, rate of progression, severity of motor and non- motor symptoms, extent of central and peripheral inflammation, maybe, because genetic and environmental factors act synergistically in PD pathogenesis.

A lot of recent research has focused particularly on genetic causes of PD. Although several genes have been implicated as monogenic causes of the disease, these genetic mutations are only responsible for approximately $10 \%$ of cases, indicating that the majority of $\mathrm{PD}$ is the result of a multifactorial pathogenic process [4] and environmental causes also may play a role in developing the disease. Rare (causative) and common (risk) variants associated with PD have been identified, including SNCA and LRRK2.

Some authors propose that environmental factors (pesticides and infections) increase the risk for $\mathrm{PD}$ via the immune system [5], because several of the genes associated with PD risk, function in the immune system. Peripheral immune activation and neuroinflammation in the brain contribute to neuropathology and neurodegeneration [6]. An environmental exposure that increases a-synuclein expression and/or inflammatory cytokine secretion could create a state in which dopaminergic neurons are vulnerable to immune-driven stress [5]. Increased kinase activity associated with the G2019S LRRK2 mutation may contribute to shifts in immune cell population frequencies and function [7]. In response to an exposure or experience, physiological and epigenetic regulation can occur, and the resulting cellsignaling cascades could influence disease pathogenesis [8]. The immune system's response to exposure may depend on the genetic variations or mutations of SNCA, LRRK2, 
PINK1, and MHCII. There is a gene-by-environment-byimmune- system triangle in $\mathrm{PD}$ pathogenesis [5].

So, $\mathrm{PD}$ risk can be modulated by cumulative and interactive effects of genes and exposures [4]. One study showed that gene-environmental interactions increased the OR for PD from about 1.6 at the individual level up to OR 12.6 for some combinations [4].

Environmental factors such as drinking well water, rural living, farming, exposure to agricultural chemicals, farm and industrial compounds, exposure to different metals and industrial compounds as manganese, lead, copper, iron, zinc, aluminium or amalgam have been reported to be associated with the risk of developing PD [9-11].

So far, is known that toxin exposure can promote PD by several mechanisms: oxidative stress, dopamine homeostasis, calcium homeostasis, alpha-synuclein fibrillization, mitochondrial dysfunction neuroinflammation [12].

As toxic exposures to these compounds can result in a spectrum of $\mathrm{PD}$ and related disorders, it is imperative to identify, not only their mechanisms of action, but also shared clinical patterns to further delineate the resultant disorders for improving diagnosis, preventive strategies and therapeutic interventions [12].

\section{Material and methods}

These are preliminary data of a cohort study of Moldovan patients with incident of Parkinson's disease. Diagnosis of PD was based on widely acknowledged criteria [13]. Structured interview on medical and drug history, family history of neurological and psychiatric diseases, years of education, all previous and current occupations, previous and current lifestyle habits, details regarding exposure to pesticides, and other toxins were recorded and a general neurological and medical examination conducted. Severity of parkinsonism and disability were assessed by the Modified Unified Parkinson's Disease Rating Scale (MDS-UPDRS) [14]. Patients were subclassified at baseline into three groups according to Jankovic method: tremor dominant (TD), akinetic-rigid (AR) or intermediate (IND) [15]. The groups weredefined as: (1) Toxic substances contact present (ToxSC+) - study group; and (2) absent (ToxSC-) - control group. The data analysis was performed via statistical program StatDirect, using descriptive, variation, and correlational analysis. Student's t tests or Mann-Whitney tests were used as appropriate. $\mathrm{P}$ values less than 0.05 were considered statistically significant.

\section{Results and discussion}

These are preliminary data of a cohort study of Moldovan patients with incident Parkinson's Disease (PD). The study group consisted of 111 consecutive PD patients. The mean age in the cohort was $64.87 \pm 7.69$ years. By sexes, PD patients, were distributed as follows: 48 were women (43.2\%) and 63 were men (56.8\%)).

Contact with toxic substances (ToxSC), was recorded by history taking about previous and current occupations, previous and current lifestyle habits, exposure to pesticides, and other toxins; and was found in 33 patients (29.73\%). In this study, excessive exposure to toxic environmental factors was more common in men and rural residents. Among ToxSC+ patients, 30 patients $(90.9 \%)$ were men and $3(9.1 \%)$ patients were women $(\mathrm{p}=0.000)$. Nineteen of the toxin exposed patients $(57.6 \%, \mathrm{p}=0.000)$ were rural residents. Similarly, in a meta-analysis of PD risk, RR was statistically significant for: rural living $(\mathrm{RR}=1.43 ; 95 \% \mathrm{CI}=1.22-$ 1.69), farming ( $\mathrm{RR}=1.24 ; 95 \% \mathrm{CI}=1.12-1.37)$ and wellwater consumption $(\mathrm{RR}=1.30 ; 95 \% \mathrm{CI}=1.12-1.51)$; the association between pesticide use and $\mathrm{PD}$ was statistically significant for all studies combined $(\mathrm{RR}=1.22 ; 95 \% \mathrm{Cl}=$ 1.18-1.27); and use of herbicides $(\mathrm{RR}=1.20 ; 95 \% \mathrm{CI}=1.06-$ $1.36)$ or insecticides $(\mathrm{RR}=1.32 ; 95 \% \mathrm{CI}=1.14-1.52)$ was associated with statistically significantly increased PD risk [8]. Also, in Norwegian ParkWest study, agricultural work was associated with a higher risk of PD (OR 1.75 (1.03-3.0) P 5 0.009): PD patients were more often agricultural workers than controls $(23 \%$ PD vs $14.4 \%$ of controls, P 50.026 [ $\chi 2$ test], odds ratio [OR] 1.75 [1.03-3.0]); whether patients or controls were born and raised on a farm did not affect the risk of $\mathrm{PD}$ [9].

Different toxic exposure was found in the study group: petrol intake (10 patients $(9 \%))$, diesel intake (4 patients $(3.6 \%))$, petrol + diesel intake (6 patients $(5.4 \%))$, exposure to pesticides (7 patients $(6.3 \%))$, to solvents -3 patients $(2.7 \%)$, to reinforced concrete (polystyrene) - 1 patient $(0.9 \%)$, to welding gas -1 patient $(0.9 \%)$, to freon -1 patient (0.9\%).

In a study, prevalence of parkinsonism among active male welders age 40 to 69 statewide was 977 to 1336 cases/100000 population. The prevalence of Parkinsonism was higher among welders vs age-standardized data for the general population (prevalence ratio - 10.19, 95\% CI 4.43 to 23.43). The authors concluded that the estimated prevalence of parkinsonism was higher within a sample of male welders vs the general population of males [16]. By contrast, the results of Danish Cohort Study (5867 Danish welders and 1735 non- welding metal workers exposed to welding fume from general Danish population in 1987-2008) do not support the hypothesis that welders are at increased risk for Parkinson's disease [17]. It was established that parkinsonism can occur after chronic exposure to high levels of manganese, usually above the permissible exposure limit ceiling at $5 \mathrm{mg} / \mathrm{m} 3$ total dust set by the Occupational Safety and Health Administration [18].

In the present study was found that in ToxSC+ patients, the disease began at an insignificantly younger age than in ToxSC-patients $(59.24 \pm 6.93$ vs $60.95 \pm 8.86$ years, $\mathrm{p}<0.005)$. By history taking, was established, that bradykinesia was the most often PD onset symptom in ToxSC+ patients (57.6\%, p $=0.008$ ). Applying the Jankovic method of defining PD motor phenotype, the more frequent PD phenotype in ToxSC+ patients was the akinetic-rigid one $(64.7 \%, \mathrm{p}=0.040)$.

There are interesting clinical differences between subgroups of PD patients (tremor dominant (TD) vs postural 
instability gait difficulties PIGD), provided by literature. In a study, a protective association of alcohol and smoking was only seen in postural instability gait difficulties (PIGD) akinetic-rigid PD and not in tremor dominant (TD) PD [9], may be because the underlying pathogenic mechanisms are heterogeneous, including environmental exposure.

Levodopa equivalent daily dose needed to compensate motor impairment in ToxSC+ patients was significantly higher than in the control group (659.02 \pm 232.46 vs 483.77 $\pm 355.41, \mathrm{p}=0.042$ ), as an indicator of a more severe motor disability in PD patients exposed to environmental toxic factors.

Overall, studies suggest that environmental insults may play an important role in the appearance and progression of PD pathology [19], PD onset and its clinical presentation may be due to a combination of external aggressors and individual genetic susceptibility to this aggression; and low incidence of PD suggests that gene-environment interactions play an important role in the process. According to our results excessive exposure to toxic environmental factors was more common associated with the akinetic-rigid type of Parkinson's disease - a phenotype with more severe motor impairment. And ToxSC+ patients needed higher doses of dopaminergic drugs - an indicator of a more motor impairment in this category.

\section{Conclusions}

Environmental factors may play an important role in the appearance, progression and clinical presentation of Parkinson's disease. This study, replicated that excessive exposure to toxic environmental factors is more commonly found in men and rural residents. According to the received results, toxin exposure was more frequently associated with the akinetic-rigid type of Parkinson's disease and with higher doses of dopaminergic drugs needed for motor symptoms control, indicating a higher severity of motor impairment in toxin exposed PD patients.

\section{References}

1. Von Campenhausen S, Bornschein B, Wick R, et al. Prevalence and incidence of Parkinson's disease in Europe. Eur Neuropsychopharmacol. 2005;15(4):473-490. doi: 10.1016/j.euroneuro.2005.04.007.

2. Lüth T, König IR, Grünewald A, et al. Age at onset of LRRK2 p.Gly2019Ser is related to environmental and lifestyle factors. Mov Disord. 2020;35(10):1854-1858. doi: 10.1002/mds.28238.
3. Galanaud JP, Elbaz A, Clavel J, et al. Cigarette smoking and Parkinson's disease: a case-control study in a population characterized by a high prevalence of pesticide exposure. Mov Disord. 2005;20(2):181-189. doi: $10.1002 / \mathrm{mds} .20307$

4. McCulloch CC, Kay DM, Factor SA, et al. Exploring gene-environment interactions in Parkinson's disease. Hum Genet. 2008;123(3):257-265. doi: 10.1007/s00439-008-0466-z.

5. Kline EM, Houser MC, Herrick MK, et al. Genetic and environmental factors in Parkinson's disease converge on immune function and inflammation. Mov Disord. 2021;36(1):25-36. doi: 10.1002/mds.28411.

6. Hirsch EC, Standaert DG. Ten unsolved questions about neuroinflammation in Parkinson's disease. Mov Disord. 2021;36(1):16-24. doi: 10.1002/ mds.28075.

7. Wallings RL, Herrick MK, Tansey MG. LRRK2 at the interface between peripheral and central immune function in Parkinson's disease. Front Neurosci. 2020;14:1-14. doi: 10.3389/fnins.2020.00443.

8. Breckenridge CB, Berry C, Chang ET, et al. Association between Parkinson's disease and cigarette smoking, rural living, well-water consumption, farming and pesticide use: systematic review and meta-analysis. PLoS One. 2016;11(4):1-42. doi: 10.1371/journal.pone.0151841.

9. Skeie GO, Muller B, Haugarvoll K, et al. Differential effect of environmental risk factors on postural instability gait difficulties and tremor dominant Parkinson's disease. Mov Disord. 2010;25(12):1847-1852. doi 10.1002/mds.23178.

10. Pan-Montojo F, Reichmann H. Considerations on the role of environmental toxins in idiopathic Parkinson's disease pathophysiology. Transl Neurodegener. 2014;3:1-13. doi: 10.1186/2047-9158-3-10.

11. Tanner CM, Kame F, Ross GW, et al. Rotenone, paraquat, and Parkinson's disease. Environ Health Perspect. 2011;119(6):866-872. doi: 10.1289/ ehp.1002839.

12. Caudle WM, Guillot TS, Lazo CR, et al. Industrial toxicants and Parkinson's disease. Neurotoxicology. 2012;33(2):178-188. doi: 10.1016/j. neuro.2012.01.010.

13. Postuma RB, Poewe W, Litvan I, et al. Validation of the MDS clinical diagnostic criteria for Parkinson's disease. Mov Disord. 2018;33(10):16011608. doi: $10.1002 / \mathrm{mds} .27362$.

14. [Goetz CG, Tilley BC, Shaftman SR, et al. Movement Disorder SocietySponsored Revision of the Unified Parkinson's Disease Rating Scale (MDS-UPDRS): Scale presentation and clinimetric testing results. Mov Disord. 2008;23(15):2129-2170. doi: 10.1002/mds.22340.

15. Mazakis A. Variable Expressions. I was waiting to see what you would do first. Fayetteville: University of Arkansas Press; 2020. p. 46-47.

16. Cox LA. Prevalence of parkinsonism and relationship to exposure in a large sample of Alabama welders. Neurology. 2006;66(4):616-617. doi: 10.1212/01.wnl.0000216107.19411.e8.

17. Kenborg L, Funch Lassen C, Hansen J, et al. Parkinson's disease and other neurodegenerative disorders among welders: a Danish cohort study. Mov Disord. 2012;27(10):1283-1289. doi: 10.1002/mds.25125.

18. Brenner SR. Searching for a relationship between manganese and welding and Parkinson's disease. Neurology. 2006;66(3):458-459. doi: 10.1212/01. wnl.0000209200.33974.b4.

19. Ball N, Teo WP, Chandra S, et al. Parkinson's disease and the environment. Front Neurol. 2019;10:218. doi: 10.3389/fneur.2019.00218.

Author's ORCID iD and academic degrees. Lilia Rotaru, MD, PhD, Associate Professor - https://orcid.org/0000-0002-5340-5234

Author's contribution. AC conceptualized the idea, conducted literature review, wrote the manuscript, revised and finalized the text.

Funding. This study was supported by Diomid Gherman Institute of Neurology and Neurosurgery. The trial was the author's initiative. The author is independent and takes responsibility for the integrity of the data and accuracy of the data analysis.

Ethics approval and consent to participate. The research project was approved by the Research Ethics Committee of Diomid Gherman Institute of Neurology and Neurosurgery (protocol No 1, 27.02.2020).

Conflict of Interests. No competing interests were disclosed. 